\title{
Rotating Elements Fault Diagnosis Method Based on Stochastic Resonance with Triple-well Potential System of Genetic Algorithm
}

\section{Liu ZiWen}

Donghua University https://orcid.org/0000-0003-0702-5190

Bao JinSong ( $\nabla$ bao@dhu.edu.cn )

Xiao Lei

Donghua University

Wang BoBo

Donghua University

\section{Original Article}

Keywords: Genetic algorithm, Stochastic resonance, Fault detection, Three-potential nonlinear system, Rotating elements

Posted Date: April 2nd, 2020

DOI: https://doi.org/10.21203/rs.3.rs-20566/v1

License: (1) This work is licensed under a Creative Commons Attribution 4.0 International License. Read Full License 


\section{Title page}

\section{Rotating Elements Fault Diagnosis Method Based on Stochastic Resonance with Triple-well Potential System of Genetic Algorithm}

Zi-Wen Liu, born in 1996, master of Mechanical Engineering, Donghua University, research direction is PHM.

E-mail:2180707@mail.dhu.edu.cn

Jin-Song Bao, born in 1977, Ph.D., Professor, worked at the School of Mechanical Engineering of Donghua University. He is currently the director of the Institute of Mechanical Engineering at Donghua University, and the director of the Institute of Intelligent Manufacturing.

Lei Xiao, born in 1988, is an assistant professor at School of Mechanical Engineering, Donghua University, China, since 2018. Her research interests include the key techniques in condition-based maintenance (CBM), such as fault detection, remaining useful life prediction and the joint optimization of maintenance and production scheduling/spare part purchasing. She got her PhD degree from Chongqing University in 2016 and then worked as a postdoctoral researcher at Shanghai Jiao Tong University.

E-mail: leixiao211@dhu.edu.cn

Bo-Bo Wang, born in 1997, master of Mechanical Engineering, Donghua University, research direction is PHM.

E-mail: wbb392797665@sina.com

\section{Corresponding author: Jin-Song Bao E-mail: bao@dhu.edu.cn}




\title{
Rotating Elements Fault Diagnosis Method Based on Stochastic Resonance with Triple-well Potential System of Genetic Algorithm
}

\author{
Zi-wen Liu ${ }^{1}, \bullet$ Jin-Song Bao ${ }^{1} \cdot$ Lei Xiao $^{1} \bullet$ Bo-Bo Wang $^{1}$
}

Received June xx, 201x; revised February xx, 201x; accepted March xx, 201x

(C) Chinese Mechanical Engineering Society and Springer-Verlag Berlin Heidelberg 2017

\begin{abstract}
In engineering applications, the fault signal of rotating elements is easily submerged in the background noise. In order to solve this problem, a rotating body fault diagnosis method based on stochastic resonance with triplewell potential system of genetic algorithm is proposed. In this method, the signal-to-noise ratio(SNR) of the Triplewell potential system is used as the fitness function of the genetic algorithm, and several parameters of the system are optimized at the same time, which effectively improves the feature extraction effect of the weak fault of the rotating body. The results of simulation and engineering experiments show that this method has better detection effect than bistable stochastic resonance method, and can effectively detect the fault signal submerged by noise, which has a good engineering application prospect.
\end{abstract}

Keywords: Genetic algorithm - Stochastic resonance • Fault detection - Three-potential nonlinear system • Rotating elements

\section{Introduction}

In practical engineering applications, there is a lot of noise in the environment, which makes it difficult to extract the weak fault signal of the rotating body. Therefore, how to realize the weak fault signal extraction under the background of strong noise has become a key problem in the field of fault detection. Most of the existing weak fault extraction

Jin-Song Bao bao@dhu.edu.cn methods realize weak signal extraction by eliminating noise, such as empirical mode decomposition[1], local mean decomposition[2], wavelet transform[3], principal component analysis[4], etc. However, while reducing noise, these methods also cause loss of useful signals. Stochastic resonance is different from the traditional denoising method. Its principle is to convert some noise energy into useful signal energy, so as to increase the signal-to-noise ratio and improve the detection ability of weak fault signal.

Stochastic resonance(SR) is proposed Benzi et al[5]. Then it is widely used in signal processing and detection[6]-[10]. Yang et al [11] proposed an application of stochastic resonance in early fault detection for intermediate gearbox of helicopter, which realizes the extraction of the early weak fault feature of gear. Tan et al. [12] combines the weighted kurtosis index with the variable-scale stochastic resonance, proposes an adaptive stochastic resonance detection algorithm for impact signals, and realizes the detection of weak impact signals. LI et al. [13] developed a weak signal detection based on step-changed stochastic resonance mothed, which can adapt to weak signal detection in both small and large parameters. Wang et al. [14]proposed a multi-parameter synchronous optimization adaptive stochastic resonance algorithm based on genetic algorithm and applied it to fault detection of rolling bearing. Hao et al. [15] proposed a mechanical fault diagnosis method based on cascade bistable stochastic resonance and multifractal, which can effectively eliminate the high frequency noise and enhance the energy of the low frequency signal, and the fractal dimension obtained from this method is more accurate, and the nonlinear characteristics of the mechanical

College of Mechanical Engineering, DongHua University, Shanghai 201620, China 
vibration signal can be measured more accurately, so as to achieve the purpose of mechanical fault diagnosis. Shi et al. [16] developed a fault diagnosis method for rotating machinery based on AMD and stochastic resonance.

In these above works and most of the current researches based on stochastic resonance mostly adopt the classical bistable stochastic resonance. Although it can also improve the signal-to-noise ratio to a certain extent and achieve weak fault signal detection. However, when the signal-to-noise ratio is used as the evaluation index, it cannot reflect the strengthening effect of a particular fault feature in the composite signal[17]. In this paper, rotating elements fault diagnosis method based on stochastic resonance with triplewell potential system of genetic algorithm is adopted. Taking the envelope signal with fault characteristic frequency as the input signal to solve the problem that the effect of bistable stochastic resonance on modulation signal enhancement is not ideal, the system parameters were optimized by genetic algorithm to extract the extraction of rotating body fault characteristics under strong noise background

\section{Stochastic Resonance with Three-well Potential Function}

\subsection{The Potential Function}

In the presence of random white noise and external force, the motion of a Brownian particle in a stochastic resonance system is subject to Langevin equation as:

$$
\begin{array}{r}
x^{\prime}(t)=U^{\prime}(x)+q(t)+E(t), \\
<E(t)>=0, \\
<E(t), E(0)>=2 D \xi(\tau),
\end{array}
$$

where $U(x)$ is the potential function of the triple-well potential nonlinear system, $x$ is the system output. $q(t)$ is the input signal, $t$ is the time variable. $E(t)$ is the Gaussian white noise with the intensity $D$ and the mean value of 0 . $D$ stands for noise intensity and $\mathrm{t}$ stands for time. $\xi(\tau)$ stands for noise single.

The potential function is as:

$$
U(x)=\frac{1}{2} a x^{2}+\frac{1}{4} b x^{4}+\frac{1}{6} c x^{6},
$$

where $a, b$, and $c$ are system parameters. These parameters are interactive and affect the steady-state point of the nonlinear system. In this paper, we set $a$ and $c$ are positive values. $b$ is set to a negative value.

Let $b^{2}=16 a^{2} c / 3, \quad b<0$, the potential function is shown in Fig.1. 0 represents the coordinate origin, $x_{1}$, $x_{2}, x_{3}$ represents the abscissa of the three steady-state points in the nonlinear system, and $\Delta U$ represents the potential well height.

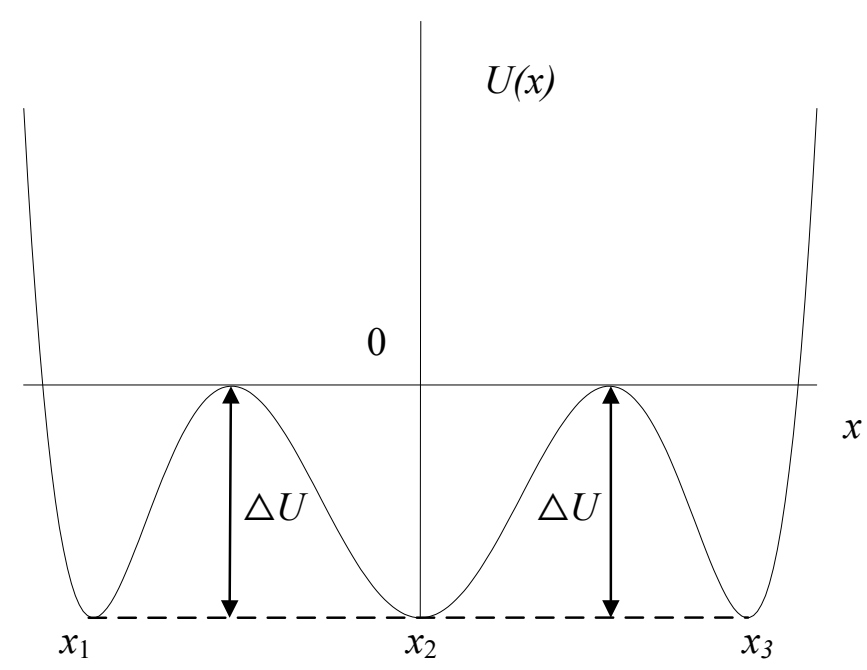

Figure1 illustration of three potential well function model

$x_{1}, x_{2}$ and $x_{3}$ can be calculated as:

$$
\begin{array}{r}
x_{1}=-\left(\frac{a+\sqrt{b^{2}-4 a c}}{2 c}\right)^{\frac{1}{2}}, \\
x_{2}=0, \\
x_{3}=\left(\frac{a+\sqrt{b^{2}-4 a c}}{2 c}\right)^{\frac{1}{2}}, \\
\Delta U=\frac{1}{12 c^{2}}\left(b^{2}-4 a c\right)^{\frac{3}{2}},
\end{array}
$$

In Eq (1), when the driving signal, noise and system reach a synergistic effect, noise plays a positive role in the nonlinear triple-well potential system, transforming part of the noise energy into signal energy, thus enhancing the signal energy. Assuming that the signal energy is small, in the absence of noise, the particle stays in any of the three potential wells. The system cannot make the particle to transit from one potential well into another. By applying a periodic force. The particle can cross the barrier height of $\Delta U$, then the system forms a continuous motion in the range 
of three potential wells, which is stochastic resonance phenomenon. It can be seen that the phenomenon of stochastic resonance of the system is related to the barrier height $\Delta U$ which is determined by the system parameters $a$, $b, c$. If the barrier height is too high, the particle needs more energy to jump from one well into another. Otherwise, if the barrier is low, it's easy for a particle to jump. Since the system parameter $b$ is determined by the system parameters $a, c$, only parameters $a, c$ are optimized in this paper.

\subsection{Parameter Optimization Based on Genetic Algorithm}

Genetic algorithm(GA) was first proposed by professor Holland from the university of Michigan[18]-[21]. it is a computational model that simulates the natural selection of Darwinian biological evolution and the biological evolution process of genetic mechanism. it is a method to search for the optimal solution by simulating the natural evolution process. Genetic algorithm is not easy to fall into local optimality, has good global search ability and fast convergence speed.
The optimization procedure of GA is shown in Fig.2.

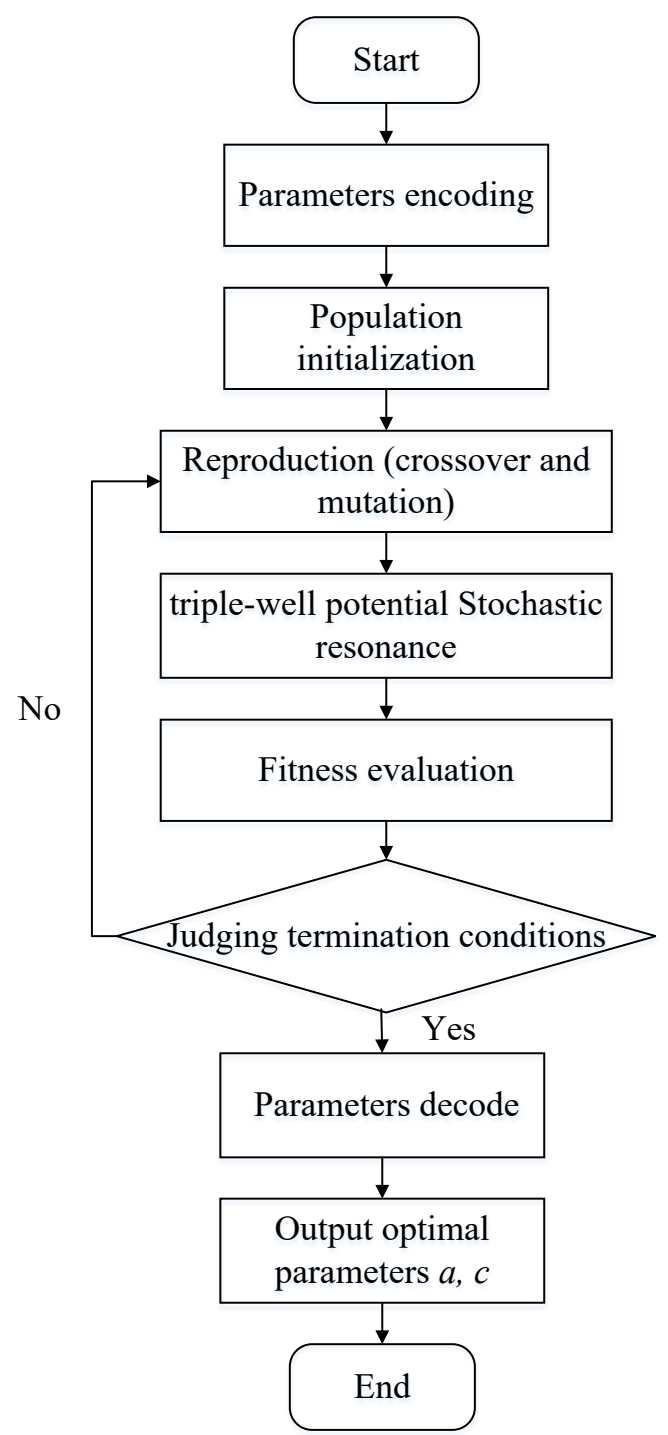

Figure 2. Optimization process of parameters $a$ and $c$ by

GA.

Step1 Parameters Encoding

Parameters a and $\mathrm{c}$ are encoded and initialized by binary.

Step2 Population initialization.

The initial population is generated randomly.

Population with larger size $M$, the length of the chromosome is $\mathrm{L}$, the number of times the population has evolved is $\mathrm{K}$.

Step3 Offspring individual screening 
The rules of roulette are used to select the superior individuals of the parents. According to a certain rule, the crossover rate $p_{a}$ and mutation rate $p_{b}$ produce a new generation of individuals through crossover and mutation.

\section{Step4 Fitness evaluation}

In this paper, the SNR of the output is used as the fitness function. SNR is widely used in the system parameter optimization. The specific formula is as[22-25]:

$$
S N R=10 \lg \frac{S\left(f_{0}\right)}{N\left(f_{0}\right)},
$$

In Eq (9), $S\left(f_{0}\right)$ represents the signal power; $N\left(f_{0}\right)$ is the average power of the same background noise.

The discrete Fourier transform is perfo rmed on the input signal $z(i)$ and output signal $l(i)$, the discrete input sequence and the output sequence are obtained as $Z(o)$ and $L(o)$, respectively.

$$
\begin{aligned}
& Z(o)=\sum_{i=0}^{I-1} z(i) e^{-\frac{2 \pi o i}{I}}, \\
& L(o)=\sum_{i=0}^{I-1} l(i) e^{-\frac{2 \pi o i}{I}},
\end{aligned}
$$

Let the peak spectrum of frequency $f_{0}$ equals to $k_{0}$, then $f_{0}=\left(k_{0} /(I-1)\right) f_{s}$ established, where $f_{s}$ is the sampling frequency, and $S\left(f_{0}\right)=\left|L\left(k_{0}\right)\right|^{2}$ is obtained. $I$ indicates the length of the sampling sequence, and $i$ is the natural number between 0 and $I$. The average power of the noise is the average power of the spectrum at $k_{0}-Z, \ldots, k_{0}-1$, $k_{0}+1, \ldots, k_{0}+Z$ near $k_{0}(Z$ is an integer selected according to the size of $I$ and $f_{s}$ ).

$$
N\left(f_{0}\right)=\frac{1}{2 Z} \sum_{j=1}^{Z}\left(\left|L\left(k_{0}-j\right)\right|^{2}+\left|L\left(k_{0}+j\right)\right|^{2}\right),
$$

where $j$ is a natural number from 1 to $Z$.

\section{Step5 Stop criterion}

Judge whether the convergence condition of the algorithm is satisfied. If so, the optimization process will be ended and the optimal parameters will be output. Otherwise, back to step 3.

\section{Simulation Verification}

In this paper, genetic algorithm is used to automatically adjust system parameters a and c, and the optimal output of the adaptive triple-well potential system is obtained. Simulation experiments are used to prove the effectiveness of this method.

A cosine signal $q(t)=A \cos (2 \pi f t)$ is provided, where in the signal amplitude $A=0.5$ and the signal frequency $f$ $=0.003 \mathrm{~Hz}$. Add Gaussian white noise with a mean of 0 and noise intensity $D=1.5$ to the signal. The time domain waveform and spectrum of the input signal are shown in Figure 3. The target frequency of $0.003 \mathrm{~Hz}$ cannot be found from the figure.

The genetic algorithm is now used to process the signal: the population size is 100 , and the maximum the precision is 0.001 , the number of iterations is 100 , the crossover rate $=0.6$, the variation rate $=0.1$, and minimum positions of $a$, $c$ are set to 1000 and 0 . the precision is 0.001 , the number of iterations is 100 , the crossover rate $=0.6$, the variation rate $=0.1$. The number of sampling points is $N=5000$, the sampling frequency $f_{s}=5 \mathrm{~Hz}$, and the optimization is $a=2.43, c=7.98$. An example is obtained in which the signal is subjected to an output response through a stochastic resonance system and analyzed in time domain and frequency domain. The time domain waveform and spectrum after the signal passes through the triple-well potential Stochastic resonance system are shown in Figure 4. The obvious spectrum can be found in the figure, which indicates that the method can detect the weak periodic component in the background noise well.
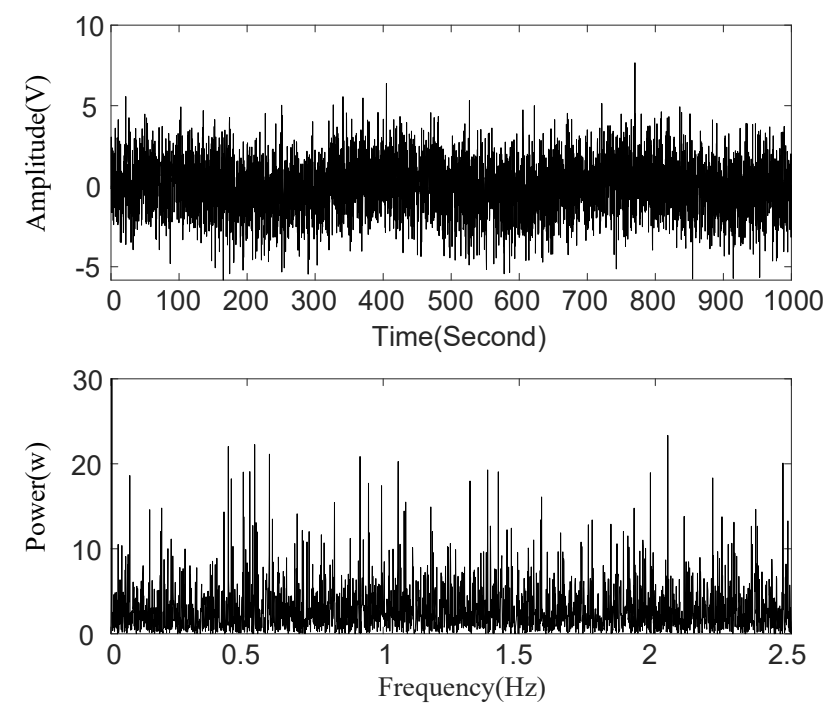

Figure 3 Simulation experiment time domain and frequency domain results 

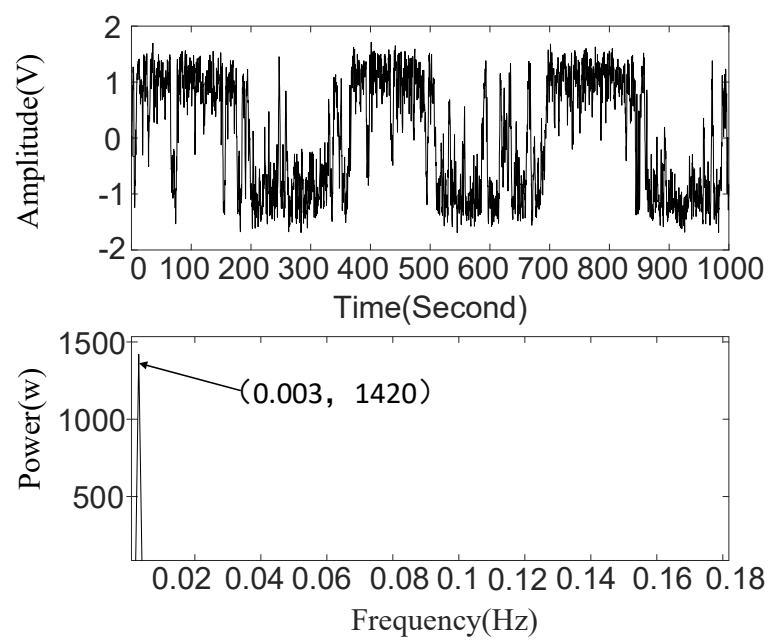

Figure 4 Time and frequency domain results of triple-well potential stochastic resonance simulation experiment

As shown in Fig.3, the time domain map and the spectrum of the original signal are disordered, and the signal frequency cannot be detected. However, the time domain diagram after the triple-well potential stochastic resonance system has a certain periodicity. At this time, the amplitude is 1420 , and the signal frequency is obvious, which is easy to be found.

\section{The engineering application}

In this section, bearing fault detection is taken as an example to verify that the above method is feasible. This section uses the relevant data published by the experimental platform of CWRU (case western reserve university). The bearing type used in the experiment is 6205-2RS JEM SKF and its specific parameters are as follows: rolling element diameter $d=0.3126$ inches, pitch diameter $D=1.573$ inches, number of balls is 9 , contact angle $r=0^{\circ}$. The input shaft speed is $1797 \mathrm{r} / \mathrm{min}$. The sampling frequency is 12000 for 1 seconds. The fault frequency of the bearing can be obtained by the NICE parameter calculation: outer ring fault $107.3 \mathrm{~Hz}$, inner ring fault $162.2 \mathrm{~Hz}$, ball fault $141.5 \mathrm{~Hz}$. The original signal time domain map and frequency domain map are shown in Fig.5.

The triple-well potential stochastic resonance setting parameters are as follows: the population number is 100 , the maximum number of iterations is 120 . After genetic algorithm $, a=1.25, c=90.6$. It can be seen from Fig.7, that the spectrum has the largest amplitude at $161.7 \mathrm{~Hz}$. Considering the rounding error, the test result is related to the fact that the bearing inner ring is faulty.
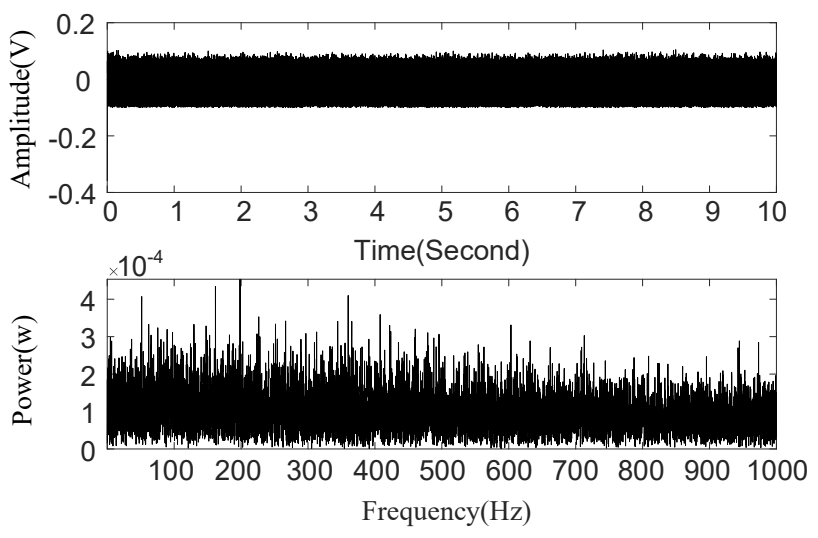

Figure 5 Time domain and frequency domain diagram of the original signal
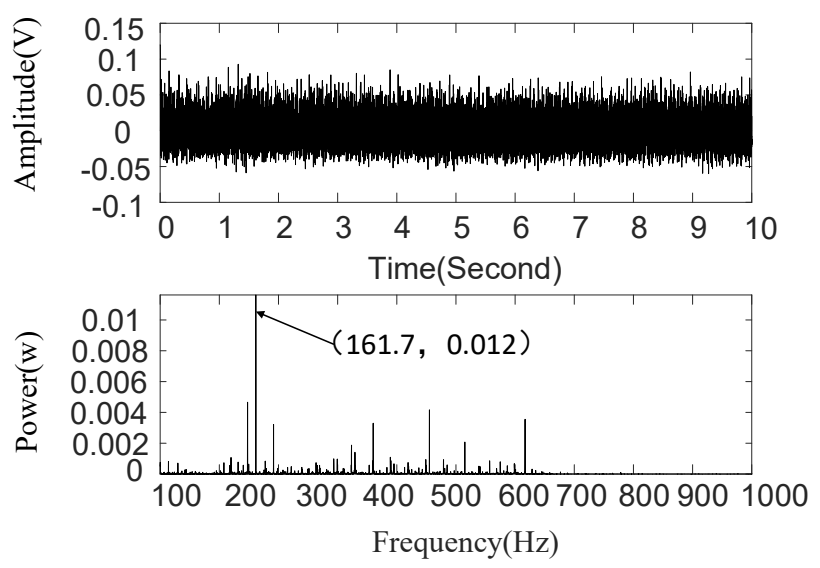

Figure 6 the time and frequency domain diagrams of the triplewell potential function Stochastic resonance
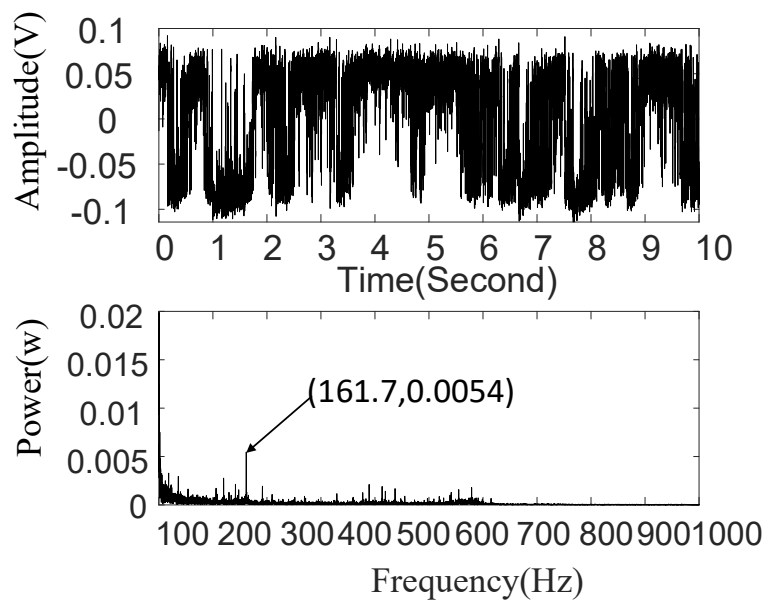

Figure 7 Time-domain and frequency-domain diagrams of the bistable stochastic resonance

For comparison, we used genetic algorithm to optimize the parameters of the bistable stochastic resonance system, and got $a=37.2, b=792.3$. The results are shown in fig.6. 
Comparing Fig.5, Fig.6 and Fig.7, it can be found that the time domain signal and the frequency domain signal of the original signal are in a mess and the frequency of the fault signal cannot be found. At the same time, the triple-well potential stochastic resonance and the bistable stochastic resonance can detect the target frequency, but the amplitude of the triple-well potential stochastic resonance is 0.012 which is much higher than 0.0054 for bistable stochastic resonance. Therefore, it can be determined that the triple-well potential stochastic resonance method performs better than traditional methods in detecting weak features.

\section{Conclusions}

(1) Rotating elements fault diagnosis method based on stochastic resonance with triple-well potential system of genetic algorithm is proposed, the system parameters a and $\mathrm{c}$ were optimized by genetic algorithm, and then the optimized stochastic resonance was used to process the fault signal of rotating elements with noise, and its spectrum was obtained to judge whether there was fault frequency in the extracted signal, and then to judge whether there was fault.

(2) By analyzing and processing the fault data of rolling bearing signal with inner ring fault, it is proved that the method proposed in this paper can extract fault characteristic frequency, which indicates that this method can realize fault diagnosis of rotating machinery and has certain practical engineering application value.

\section{Declaration}

\section{Acknowledgements}

The authors sincerely thanks to Professor Bao of DongHua University for his critical discussion and reading during manuscript preparation.

\section{Funding}

Supported by National Natural Science Foundation of China (51705321), the Fundamental Research Funds for the Central Universities (2232019D3-29), the China Postdoctoral Science Foundation (2017M611576) and the Fundamental Research Funds for the Central Universities (2232017A-03) ; Shanghai industrial internet innovation and Development Project(2018-GYHLW-01003); Energy intelligent management application platform project based on artificial intelligence(2018-RGZN-02055)

\section{Availability of data and materials}

The datasets generated and/or analysed during the current study are available in the [CWRU] repository,[https://csegroups.case.edu/bearingdatacenter/pa ges/download-data-file]

\section{Authors' contributions}

The author' contributions are as follows: Jin-Song Bao was in charge of the whole trial; Zi-Wen Liu wrote the manuscript; Lei Xiao and Bo-Bo Wang assisted with sampling and laboratory analyses.

\section{Competing interests}

The authors declare no competing financial interests.

\section{Consent for publication}

Not applicable

Ethics approval and consent to participate

Not applicable

\section{References}

[1] Y Yang, D J Yu, S J Cheng, et al. Application of emprical mode decomposition (EMD)in roller bearing fault diagnosis. Journal of Human University(Natural Sciences),2003(05):25-28. (in Chinese)

[2] S J Cheng, Y Yang, J D Yu. The local mean decomposition method and its application to gear fault diagnosis . Journal of Vibration Engineering,2009,22(01):76-84. (in Chinese)

[3] H Ye, G Z Wang, C Z Fang. Application of wavelet transform to fault detection . Acta automatica sinica,1997(06):18-23. (in Chinese)

[4] Z Z Wei. Feature extraction based on kernel principal component analysis. Journal of GuangXi university of technology, 2006(04):2731. (in Chinese)

[5] BENZI R, SUTERA A, VULPIANI A. The mechanism of stochastic resonance. Journal of Physics.1981,14 (11): L453-L457

[6] C Nicolis,. Stochastic resonance in multistable systems: The role of intermediate states. Physical Review E, 2010, 82(1):011139.

[7] S Saikia, A M Jayannavar, M C Mahato. Stochastic resonance in periodic potentials. Physical Review E, 2011, 83(6):061121.

[8] F Long, W Guo, D C Mei. Stochastic resonance induced by bounded noise and periodic signal in an asymmetric bistable system. Physica A: Statistical Mechanics and its Applications, 2012, 391(22):5305--5310.

[9] S Lu, Q He, F Kong. Stochastic resonance with Woods-Saxon potential for rolling element bearing fault diagnosis. Mechanical Systems and Signal Processing, 2014, 45(2):488-503.

[10] D S Schwarzkopf, J Silvanto, G Rees. Stochastic Resonance Effects Reveal the Neural Mechanisms of Transcranial Magnetic Stimulation. Journal of Neuroscience, 2011, 31(9):3143-3147.

[11] D X Yang, N Q Hu, Y G Yang, et al. application of stochastic resonance in early fault detection for intermediate gearbox of helicopter. Journal of Vibration Engineering, 2004(02): 85-88. (in Chinese)

[12] J Y Tan, X F Chen, Z J He. Impact signal detection method with 
adaptive stochastic resonance. Journal of mechanical engineering. 2010,46(23):61-67. (in Chinese)

[13] Q Li, T Y Wang, Y G Leng, et al. Weak signal detection based on step-changed stochastic resonance. Journal of TianJin University, 2006(04): 432-437. (in Chinese)

[14] J Wang, Q Zhang, L Liang, et al. Adaptive Stochastic Resonance Based on genetic algorithm with applications in weak signal detection. Journal of Xi'An JiaoTong University, 2010, 44(03): 3236. (in Chinese)

[15] Y Hao, T Y Wang, J Wan, et al. Mechanical fault diagnosis based on cascaded bistable stochastic resonance and multi-fractal. JOURNAL OF VIBRATION AND SHOCK, 2012, 31(08): 181-185. (in Chinese)

[16] P M Shi, C J Su, N Zhao, et al. Study on fault diagnosis method for rotating machinery based on adaptive stochastic resonance and AMD. ACTA METROLOGICA SINICA, 2017, 38(01): 112-116. (in Chinese)

[17] B H Jia, Y Feng, G D Yan, et al. Zhang. The influence of rotor crack on dynamic changes rule of Turbine tip clearance. Journal of Vibration, Measurement \& Diagnosis, 2016,36(06):11531160+1240. (in Chinese)

[18] W Q Cao, P Fu, X H Li. Early fault intelligent diagnosis of tool wear . Journal of mechanical engineering, 2014, 25(18):2473-2477. (in Chinese)

[19] J Tian, J Zhou, S G Wang, et al. Fault diagnosis method of inter-shaft bearing based on adaptive bistable stochastic resonance. Journal of aerospace power, 2019(10): 2237-2245. (in Chinese)

[20] J Wang, Q Zhang, G h Xu . Genetic Stochastic Resonance: A New Fault Diagnosis Method to Detect Weak Signals in Mechanical Systems. Advanced Science Letters, 2011.

[21] W Mu, G Liu, X Wang, et al. Research on stochastic resonance enhancement of X-ray images based on a genetic algorithm. Insight - Non-Destructive Testing and Condition Monitoring, 2016, 58(5): 246-250.

[22] L Xiao, T Xia, E Pan, et al. A novel weak bearing fault detection method based on vibrational resonance. 2018 Prognostics and System Health Management Conference (PHM-Chongqing). IEEE, 2018: 100-104.

[23] Y B LI, B L Zhang, Z X LIU, et al. Adaptive stochastic resonance method based on quantum particle swarm optimization. Acta Phys. 2014,63 (16): 40-47. (in Chinese)

[24] S B Jiao, P H Li, Q Zhang, et al. Multi-frequency weak signal detection method based on adaptive stochastic resonance with Knowledge-based PSO. Journal of mechanical engineering,2014,50(12):1-10. (in Chinese)

[25] S L Lu, Q B He, J Wang. A review of stochastic resonance in rotating machine fault detection. Mechanical Systems \& Signal Processing, 2019, 116(FEB.1):230-260.

\section{Biographical notes}

Zi-Wen Liu, born in 1996, master of Mechanical Engineering, Donghua University, research direction is PHM.

E-mail: 2180707@mail.dhu.edu.cn

Jin-Song Bao, born in 1977, Ph.D., Professor, Worked at the School of Mechanical Engineering of Donghua University. He is currently the director of the Institute of Mechanical Engineering at Donghua University, and the director of the Institute of Intelligent Manufacturing.

E-mail: bao@dhu.edu.cn
Lei Xiao, born in 1988, is an assistant professor at School of Mechanical Engineering, Donghua University, China, since 2018. Her research interests include the key techniques in conditionbased maintenance (CBM), such as fault detection, remaining useful life prediction and the joint optimization of maintenance and production scheduling/spare part purchasing. She got her $\mathrm{PhD}$ degree from Chongqing University in 2016 and then worked as a postdoctoral researcher at Shanghai Jiao Tong University.

E-mail: leixiao211@dhu.edu.cn

Bo-Bo Wang, born in 1997, master of Mechanical Engineering, Donghua University, research direction is PHM.

E-mail: wbb392797665@sina.com 
Figures

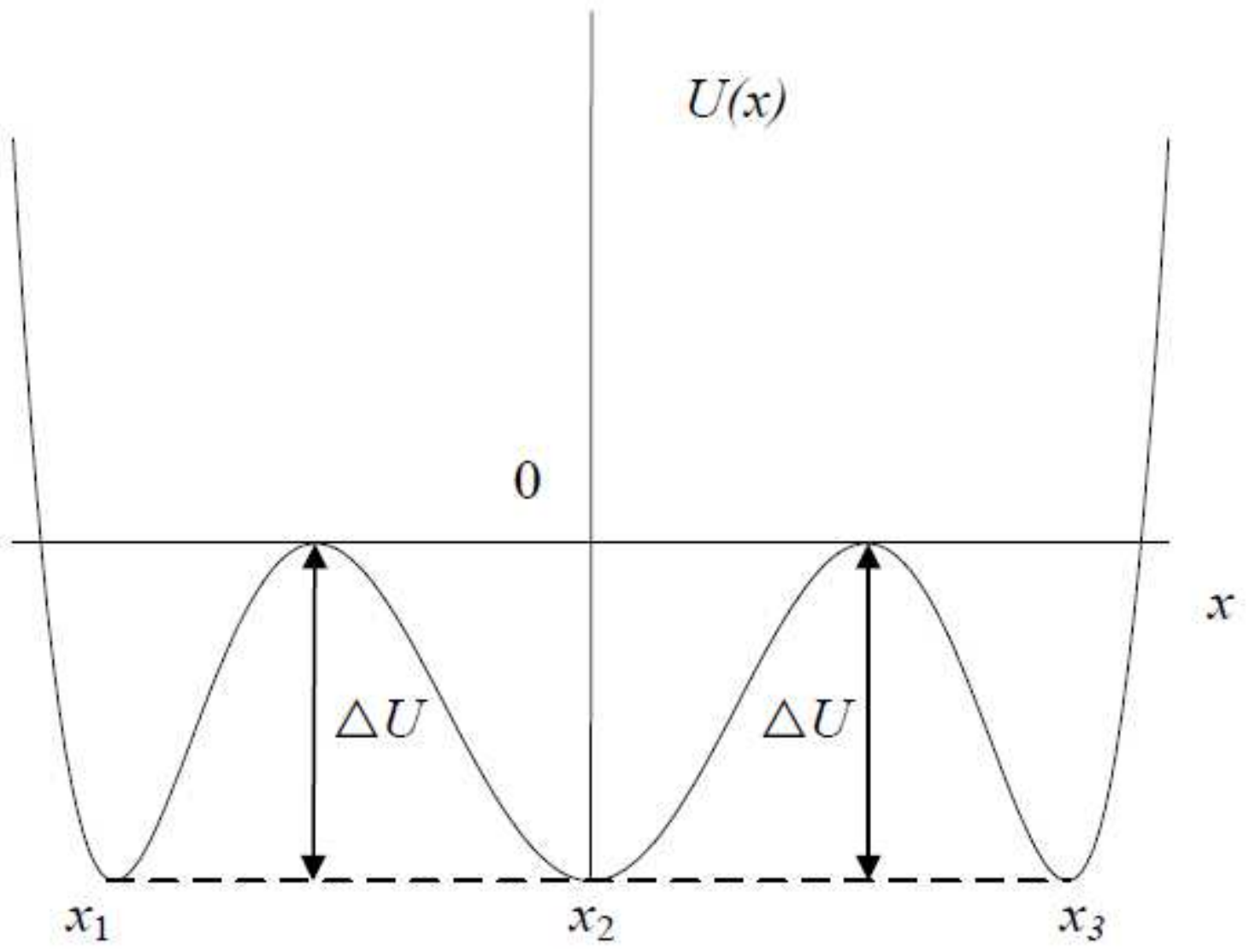

Figure 1

illustration of three potential well function model 


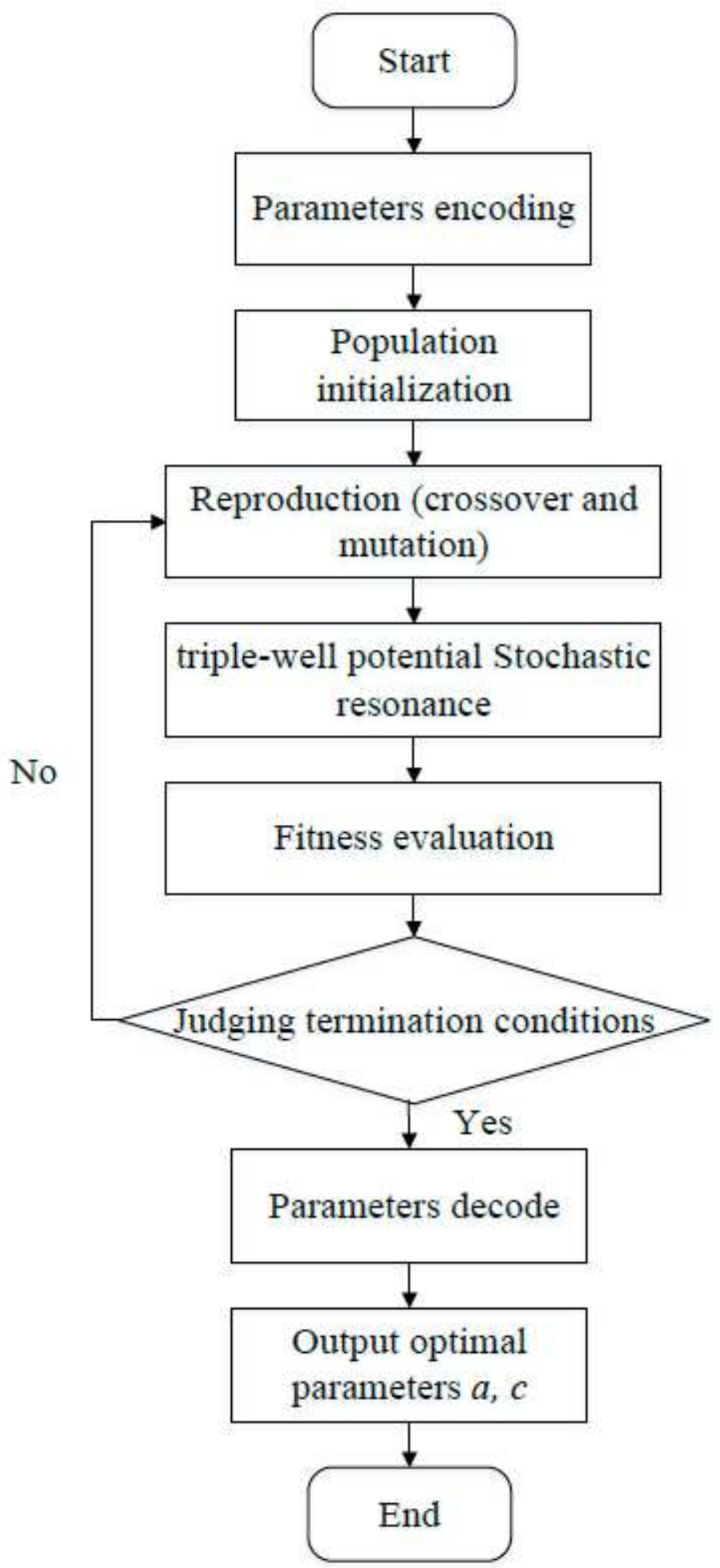

Figure 2

Optimization process of parameters a and $\mathrm{c}$ by GA. 

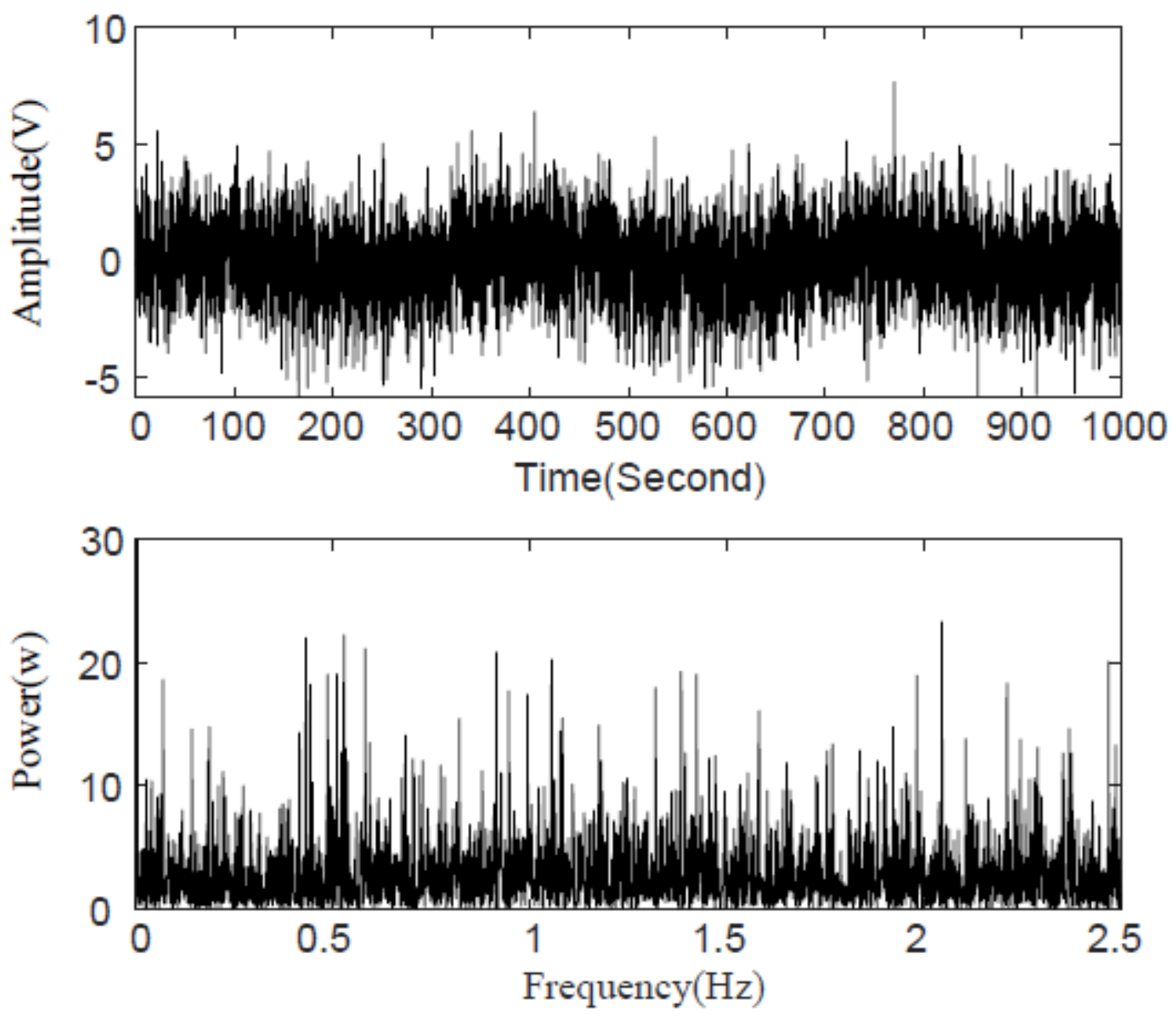

Figure 3

Simulation experiment time domain and frequency domain results 

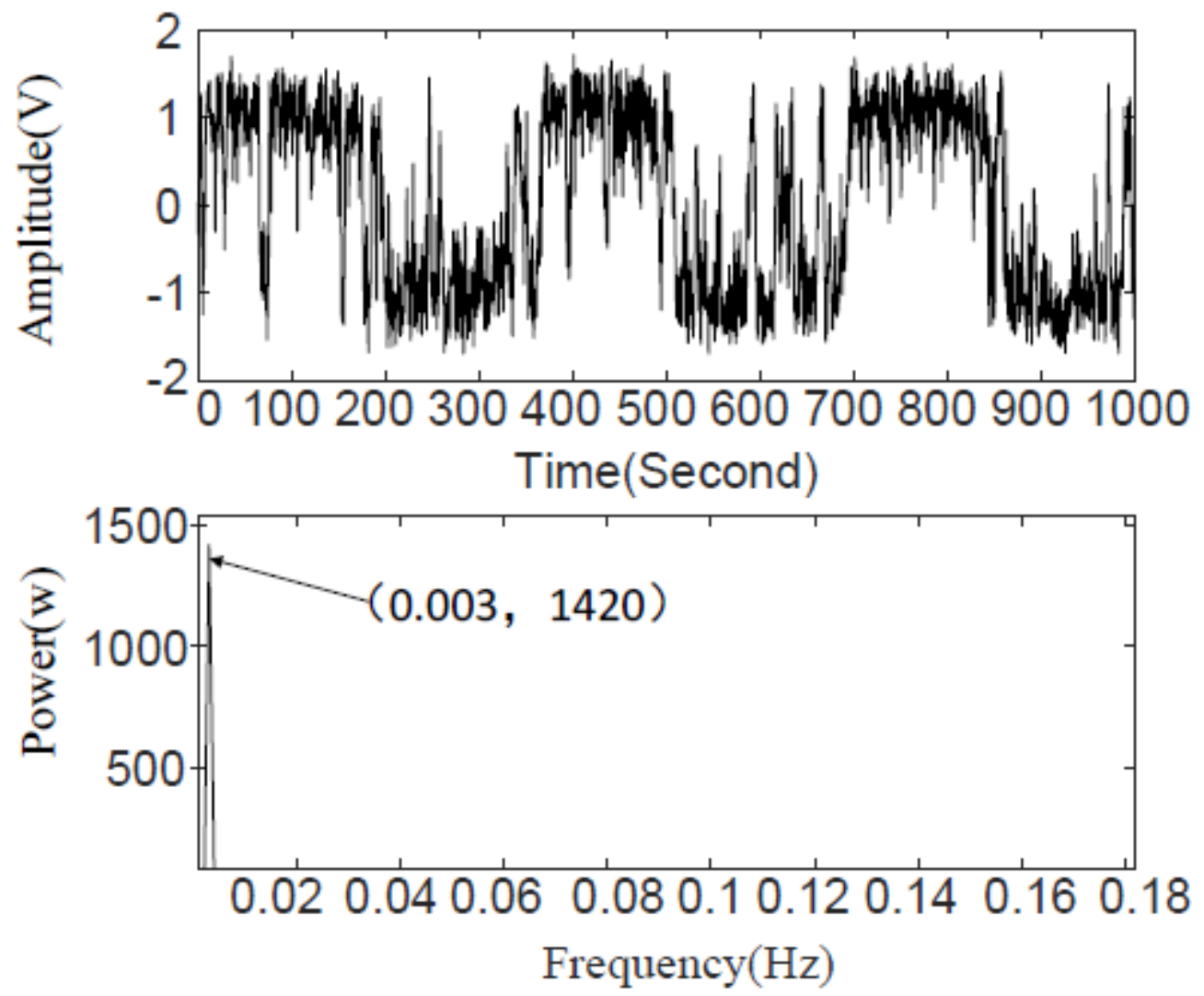

Figure 4

Time and frequency domain results of triple-well potential stochastic resonance simulation experiment
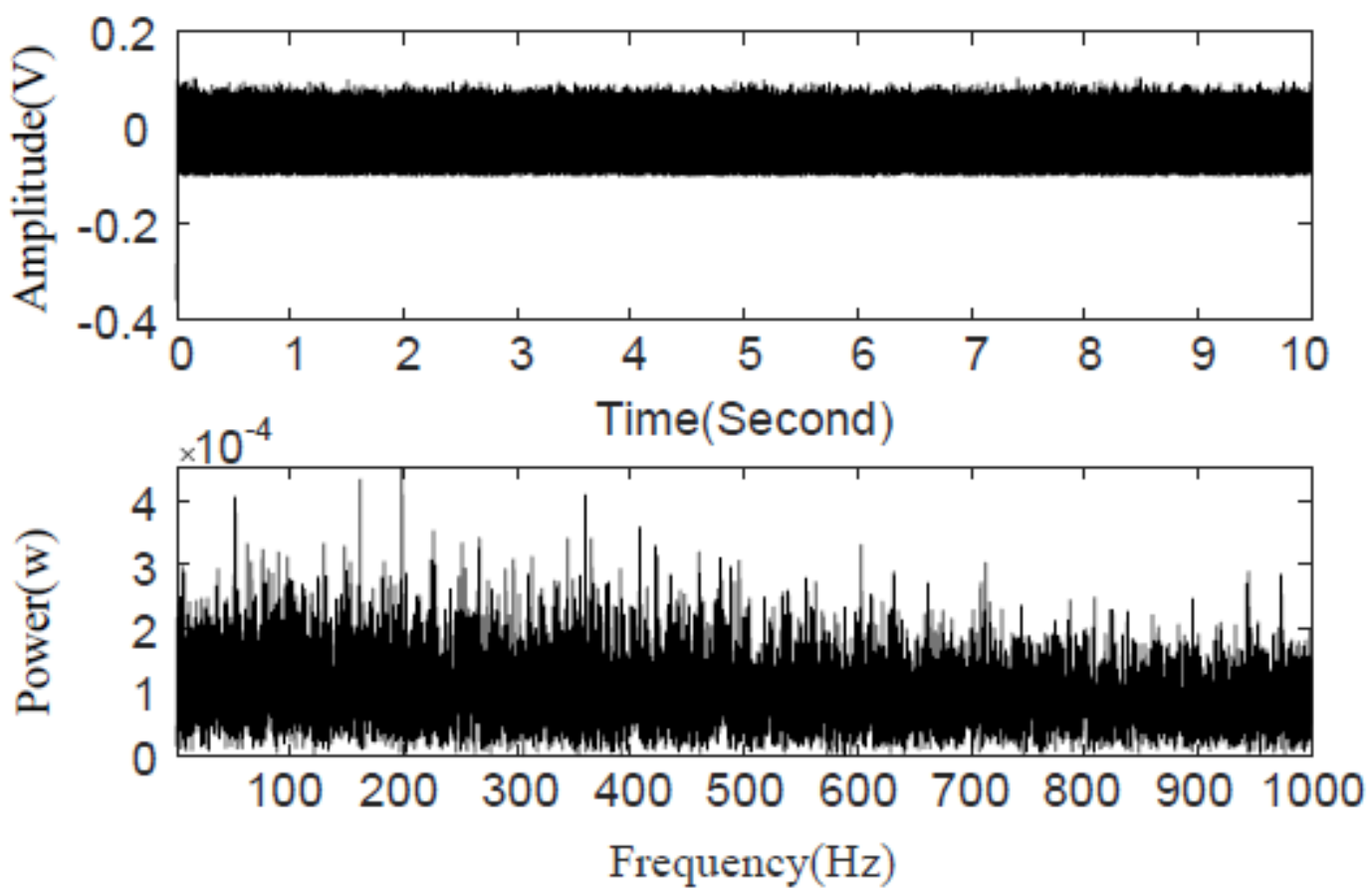

Figure 5 
Time domain and frequency domain diagram of the original signal
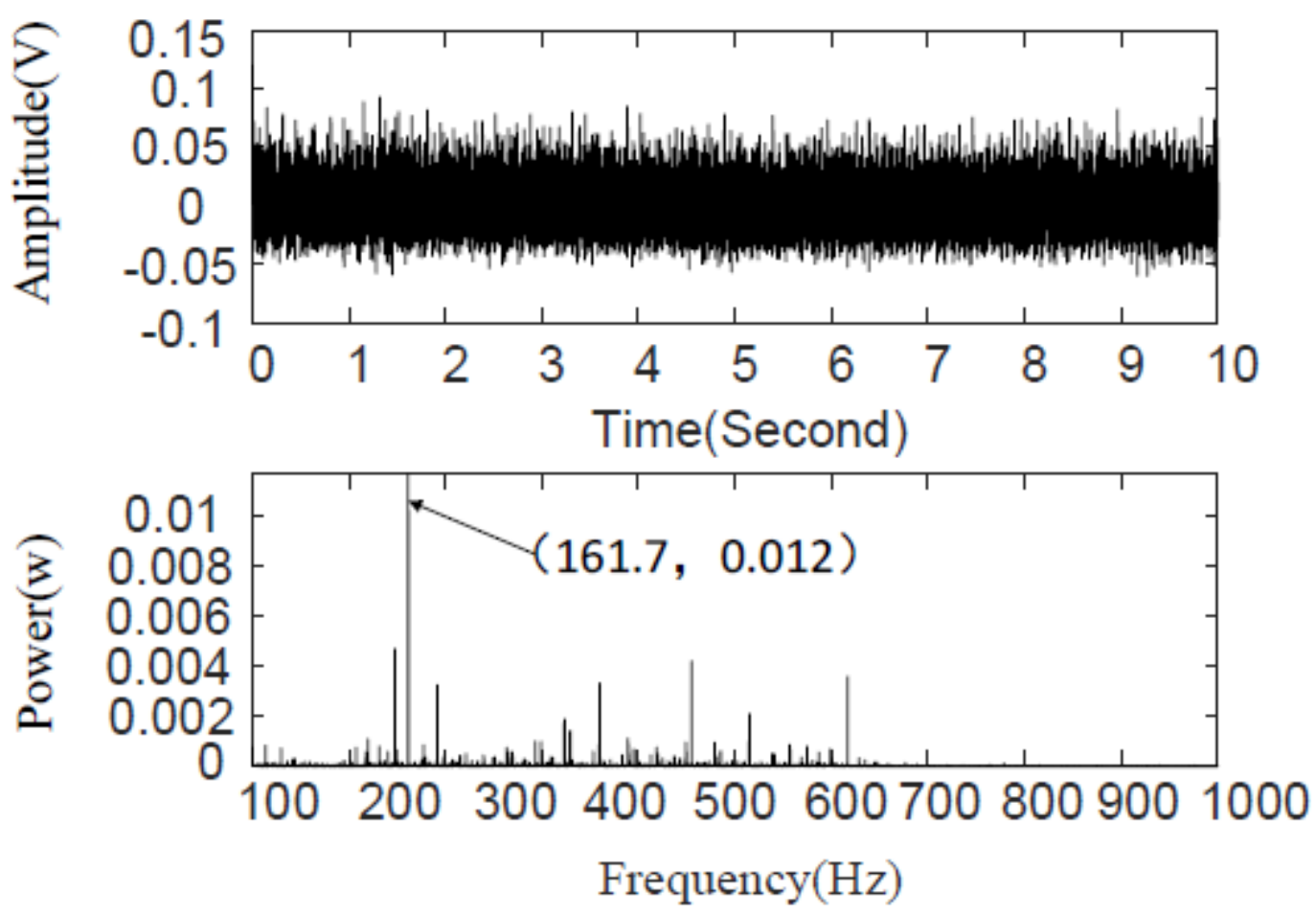

Figure 6

the time and frequency domain diagrams of the triple-well potential function Stochastic resonance
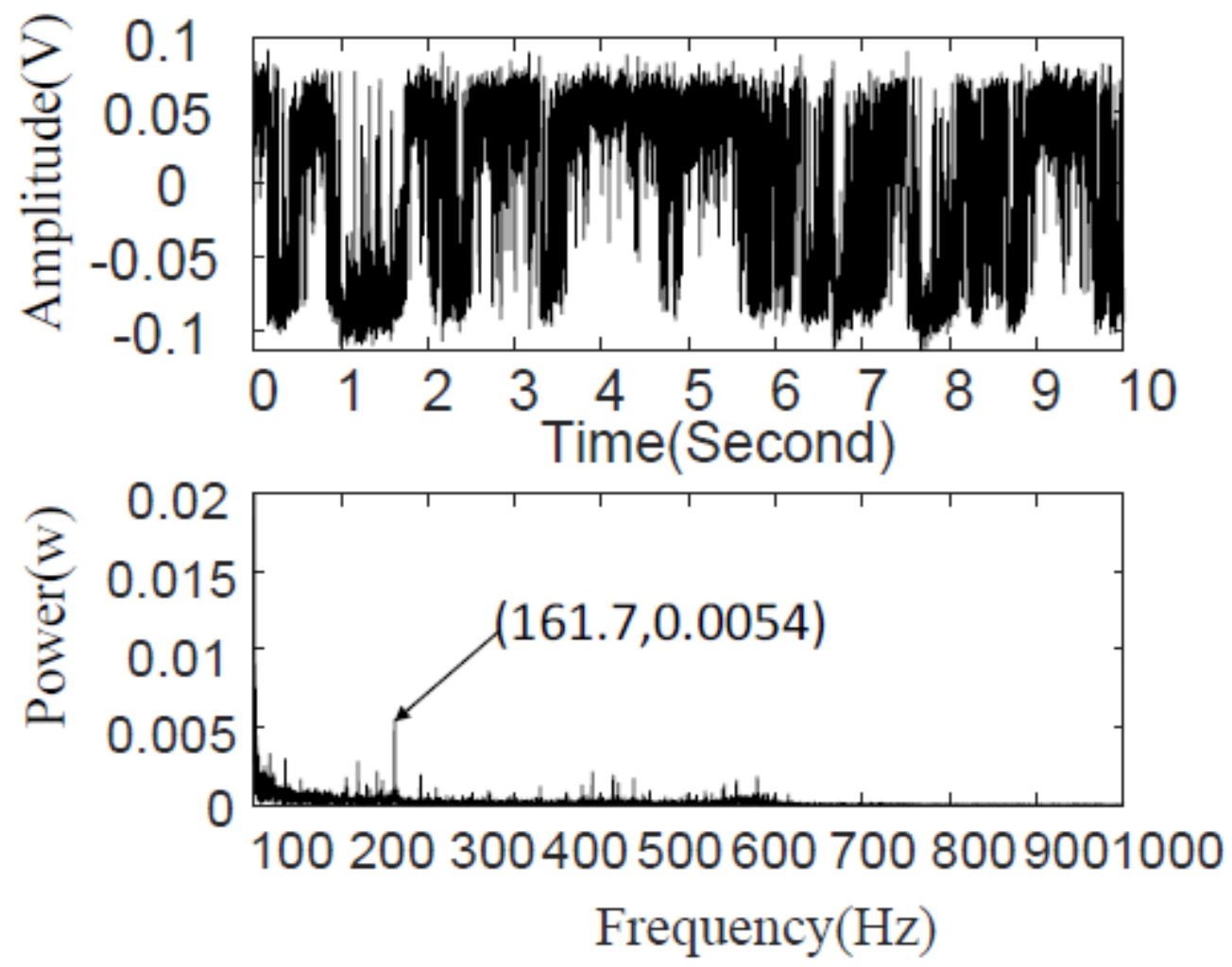

Figure 7 
Time-domain and frequency-domain diagrams of the bistable stochastic resonance 